\title{
Design and Simulation of a Highly Sensitive SPR Optical Fiber Sensor
}

\author{
Motahare Sadat HOSEINIAN $^{1}$ and Mohammad Agha BOLORIZADEH ${ }^{2 *}$ \\ ${ }^{1}$ Department of Photonics, College of Modern Sciences, Graduate University of Advanced Technology, Mahan 76311, \\ Iran \\ ${ }^{2}$ Department of Atomic and Molecular Physics, Physics Faculty, Yazd University, Yazd 89195, Iran \\ *Corresponding author: Mohammad Agha BOLORIZADEH_E-mail: mabolori@uk.ac.ir
}

\begin{abstract}
An idea of the surface plasmon resonance (SPR) has been utilized for the design of highly sensitive sensors based on the wagon-wheel fiber technology. Such sensors are sensitive to changes in the refractive index of sample analyte. In this study, a three-strut wagon-wheel structure, coated with the gold layer of nano-sized thickness, has been proposed as the SPR sensor. Finite element method is employed to simulate and tune the proposed SPR's design, which leads to a highly sensitive and multichannel plasmonic sensor with the ability for a dual reading on a single analyte or simultaneous identification of two analytes. In this design, suitable thickness values for the gold layer and core struts are determined. Sensitivities of the detector due to the first resonance peak, second resonance peak, and the difference in resonance peaks are calculated to be $1120 \mathrm{~nm} / \mathrm{RIU}$, $1540 \mathrm{~nm} / \mathrm{RIU}$, and $420 \mathrm{~nm} / \mathrm{RIU}$, respectively, when analytes are placed in all three channels of the fiber. Sensitivity of the detector with respect to the second resonant peak for analyte in Channels 2 and 3 is also found to be $1252 \mathrm{~nm} / \mathrm{RIU}$ when Channel 1 is filled with the reference. The sensitivity and resolution of the sensor increase as the refractive index of the analyte increases by almost a linear proportion. If the sensor is utilized to detect the difference in two peaks, it would substantially reduce the noise, and the best result is expected. The thicknesses of the struts and the gold layer are proper parameters to be tuned in designing the detector.
\end{abstract}

Keywords: Plasmonics; surface plasmon-polaritons; sensor; wagon-wheel fiber

Citation: Motahare Sadat HOSEINIAN and Mohammad Agha BOLORIZADEH, "Design and Simulation of a Highly Sensitive SPR Optical Fiber Sensor," Photonic Sensors, 2019, 9(1): 33-42.

\section{Introduction}

At optical frequencies, a simple metallic surface bounded by a dielectric can support surface plasmon-polaritons (SPPs). SPPs are transverse magnetically polarized optical surface waves coupled with free-electron oscillations. Surface plasmon resonance (SPR) characteristics were observed optically for the first time through an attenuated total reflection devised by Kretschmann and Raether [1], and Otto [2]. Ever since the discovery of SPR, it has been utilized in a wide variety of applications [3-9].

Resonance wavelength of plasmon depends on the refractive index of the dielectric material used to fabricate the fiber, which is in contact with the metal. A change in the refractive index of the dielectric changes the coupling between light and surface plasmon hence changing the propagation constant. This property is useful in fabricating highly sensitive

Received: 8 March 2018 / Revised: 28 May 2018

(C) The Author(s) 2018. This article is published with open access at Springerlink.com

DOI: $10.1007 / \mathrm{s} 13320-018-0508-7$

Article type: Regular 
sensors with wide industrial applications, in particular the health care industry.

Homola's review paper in 1999 [10] and his follow-up article in 2003 [11] provided an excellent overview of developments in this field for the past 20 years. In 2007, Hoa et al. [4] presented a review to focus on the progress made since 2003 towards integrable opto-electronic components suitable for SPR. In 2010, Gorman et al. [5] devised an SPR waveguide sensor based on single and dual channel design. In 2011, Cennamo et al. [12] reported the fabrication and testing of two configurations of optical sensor systems based on SPR at the interface of a liquid sample and sandwiched structures realized starting from the exposed core of a plastic optical fiber. In 2016, a novel SPR sensor based on a D-shaped, all-glass, and endless single-mode photonic crystal fiber was experimentally demonstrated by Chen et al. [13].

In 2008, Hautakorpi et al. [14] investigated the wagon-wheel fiber as a plasmonic sensor and showed that the optical loss of the Gaussian guided mode could be made very small by tuning the thickness of the dielectric layer and the refractive index resolution for aqueous analytes was $1 \times 10^{-4}$ RIU. In [15], Zhang and coworkers studied the wagon-wheel fiber as a plasmonic sensor. They calculated the influence of structural variables on sensor's performance. By changing the cladding layer thickness, sensor length, and sensing layer, they obtained the sensitivity in the range from $800 \mathrm{~nm} / \mathrm{RIU}$ to $1800 \mathrm{~nm} / \mathrm{RIU}$ over a dynamic range of 1.33 RIU to 1.36 RIU for the refractive index. They varied over layers to tune the upper limit for analytes' refractive indexes in Channels 2 and 3.

In the present study, based on numerical simulations results, we propose a wagon-wheel optical fiber that may be utilized as a plasmonic sensor with a cross section depicted in Fig. 1. The proposed sensor is capable of simultaneously detecting two analytes or yielding two distinct surface plasmon resonance peaks for the same analyte. Note should be added that a wagon-wheel structure of three struts are just an example, and the number of struts could be increased, which is not the case for this work or Zhang's and coworkers' [15].

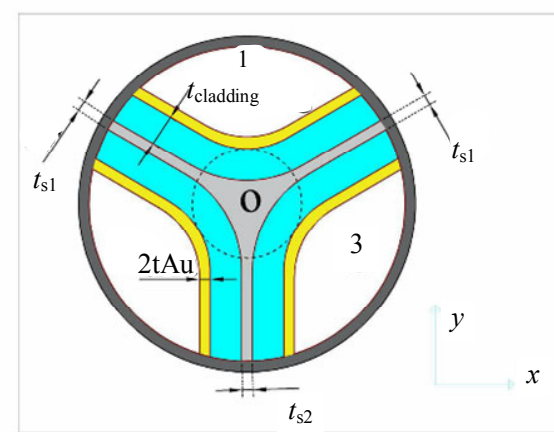

Fig. 1 Cross-sectional view of the proposed wagon-wheel fiber sensor. The outer ring has an inner diameter of $5 \mu \mathrm{m}$ and a thickness of $0.1 \mu \mathrm{m}$. The small circle around the core and the curvature of the gold layer are $0.5 \mu \mathrm{m}$ and $4 \mu \mathrm{m}$, respectively. The thickness of the cladding is taken to be $1.25 \mu \mathrm{m}$.

\section{Method and physical model}

The objective of the present design is set to maximize the sharpness and the separation of the SPR peaks to improve the sensitivity and resolution of the wagon-wheel type sensors, as shown in Fig. 1. For this design, the fixed parameters are given in the caption of Fig. 1. The refractive indexes of the ring (dark grey), the struts (light grey), and the core (light grey) are $1.46 \mathrm{RIU}$. The refractive index of the cladding (cyan) is $1.44 \mathrm{RIU}$. The difference in the refractive indexes enables mode confinement and total reflection. The refractive index of the gold layer is defined by the Drude model [16].

The proposed system is analyzed and improved upon through simulation by making use of the finite element method available from SyNA software [17, 18]. The SyNA software $[17,18]$ is a home-written software introduced in $[17,18]$. The parameters that are varied to improve the design are gold layer's thickness and/or the thickness of the core struts while best values based on the aforementioned criteria are selected. In addition, a change in the symmetry of the struts' thickness is applied to further improve the sensitivity and resolution for tuning the upper limit of the analyte's refractive index in Channels 2 and 3. 
In the present investigations, plasmon resonance frequencies of the proposed sensor are studied by making use of the finite element method and utilizing the software package SyNA. The formulation used in SyNA is based on the formalism given by Jiang et al. [19] and solves the vector equation

$$
\nabla \times \nabla \times \mathbf{E}-k_{0}^{2} n^{2} \mathbf{E}=0
$$

with the following ansatz as

$$
\mathbf{E}(x, y, z, t)=\mathbf{E}_{0}(x, y) \mathrm{e}^{\mathrm{i}(\beta z-\omega t)}=\left\{\begin{array}{l}
e_{u} \\
e_{v} \\
e_{w}
\end{array}\right\} \mathrm{e}^{\mathrm{i}(\beta z-\omega t)}
$$

where $\beta, \omega, n$, and $k_{0}(=2 \pi / \lambda)$ are the propagation constant, the angular frequency, the refractive index of the fiber's material, and the wavenumber with $\lambda$ being the free-space wavelength, respectively. Additionally, $e_{u}, e_{v}$, and $e_{w}$ are respectively the $x, y$, and $z$ components of the electric field $\mathbf{E}_{0}(x, y)$ and $\mathrm{i}=\sqrt{-1}$.

The finite element formulation of the present boundary value problem is a full vectorial case. Steps in the computational process are as follows:

(1) The Galerkin weak form of (1) in SyNA [17, $18]$ is implemented as an eigen-problem;

(2) A proper mesh is constructed for implementing the finite element formalism devised for a precise definition of the geometry and the material makeup of optical fiber's cross section;

(3) An eigen-value problem is established, and $\beta$ is the eigenvalue;

(4) Initial interpolation function with the minimal order is selected, while their order is altered to establish a computational convergence of the model;

(5) The resulting eigen-problem is solved to arrive at the propagation constant $\beta$ and the relative field amplitude.

The computational objective is to obtain the wave propagation profile of an eigen-mode in (1). In this study, $\omega$ is assumed as a known parameter, and $\beta$ is the unknown eigen-mode. The effective mode index $n_{\text {eff }}$ is defined as

$$
n_{\text {eff }}=\beta / k_{0}
$$

where it appears as a complex parameter, i.e.

$$
n_{\text {eff }}=\operatorname{Re}\left(n_{\text {eff }}\right)+i \operatorname{Im}\left(n_{\text {eff }}\right)
$$

where $\operatorname{Re}\left(n_{\text {eff }}\right)$ and $\operatorname{Im}\left(n_{\text {eff }}\right)$ stand for the real and imaginary parts of $n_{\text {eff, respectively. Furthermore, }}$ the decay in the power of a mode along the sensor's vertical symmetry axis can be evaluated as it propagates with the relation as

$$
P(z)=\mathbf{E} \cdot \mathbf{E}^{*}=P_{0} \exp \left[-2 k_{0} \operatorname{Im}\left(n_{\text {eff }}\right) z\right]=P_{0} \mathrm{e}^{-\alpha z}
$$

where $P_{0}$ (set at $1 \mathrm{~W}$ ) is the power of the eigen-mode at the plane $z=0$ and $\alpha$ is the sensor's loss. The sensor's loss due to the plasmonic resonance is related to the imaginary part of the effective mode index as follows:

$$
\alpha=2 k_{0} \operatorname{Im}\left(n_{\text {eff }}\right) .
$$

Norm of the electric field and the effective mode indexes are calculated for wavelengths over optical range from $500 \mathrm{~nm}$ to $650 \mathrm{~nm}$.

\section{Results and discussion}

In all test cases presented here, the input field is assumed to be the fundamental eigen-mode of the sensor at wavelengths in the range from $500 \mathrm{~nm}$ to $650 \mathrm{~nm}$. Based on the required values of the refractive index of the analyte in three channels, field distribution and the eigenvalues are first computed, and then the corresponding effective mode index is calculated by using (3).

Figures 2(a) and 2(b) present the real and imaginary parts of effective refractive mode index, respectively, for the $x$ - and $y$-polarized fundamental modes of the sensor versus optical wavelength. Real part of the effective refractive index shows a similar pattern as the results presented by Zhang et al. [15]. Parameters of the wagon-wheel fiber for the data presented in Fig. 2 are the ones given by the caption of Fig. 1, which are fixed throughout this work. The other parameters used to derive the results of Fig. 2 are the struts' thickness of $15 \mathrm{~nm}$ and the gold layer thickness of $27 \mathrm{~nm}$.

As it is evident from Fig. 2(a), the $x$ - and 
$y$-polarized modes are degenerate except for the regions below and above the two zero-birefringence points [20]. The two zero-birefringence points located at $568 \mathrm{~nm}$ (Point A) and $596 \mathrm{~nm}$ (Point B) dictate the occurrence of plasmonic resonance. This can be readily confirmed from the variation of the imaginary part shown in Fig. 2(b). In principle, the fundamental $x$ - and $y$-polarized modes are degenerate below $540 \mathrm{~nm}$ and above $620 \mathrm{~nm}$, while they are non-degenerate between these two wavelengths. It is important to note that the imaginary part of the effective refractive index is zero or almost zero, where the $x$ - and $y$-polarized modes are degenerate modes. Hence, the loss due to the waveguide is enhanced where the two modes are not degenerate which makes this fiber structure feasible as a sensor.

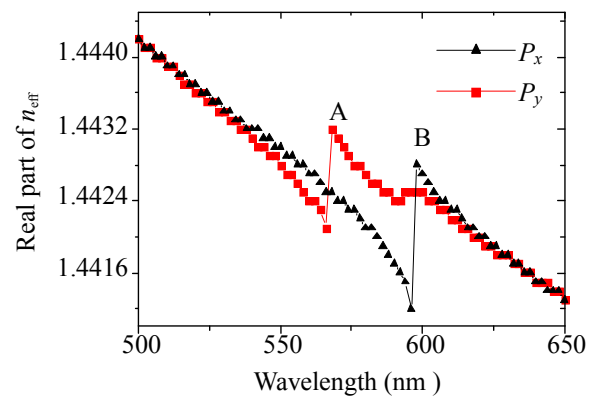

(a)

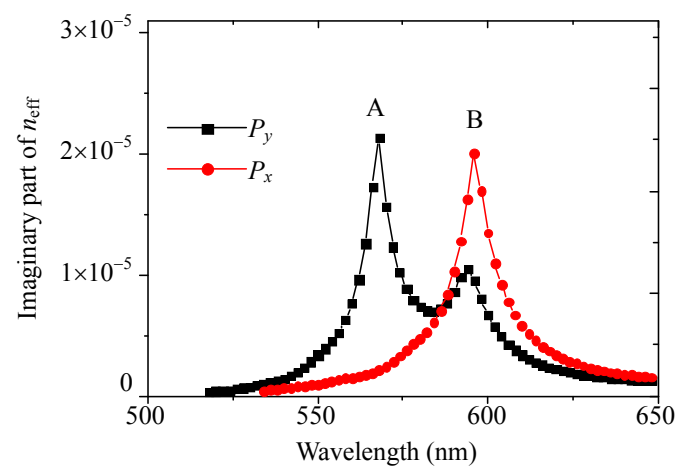

(b)

Fig. 2 The $x$ - and $y$-polarized effective refractive indexes of fundamental modes versus the input optical wavelength obtained for the wagon-wheel fiber shown in Fig. 1: (a) the real part and (b) the imaginary part.

Here, the refractive index is set to 1.33 RIU for water as the reference analyte in three channels. The formalism is set to determine two orthogonal modes, one being parallel to the symmetry axis of refractive index profile ( $y$-polarized mode) and the other being orthogonal to this axis ( $x$-polarized mode) shown in Fig. 3. The fundamental eigen-mode of the sensor with polarization in $y$-direction is selected as the resonant mode in all cases investigated here. Reasons for this choice are the strong confinement of the fundamental mode and the appearance of more than one resonant peak in $y$-polarized case of the fundamental mode. In Channels 2 and 3, the $x$ and $y$-polarized modes couple with plasmons on the gold layer's surface, while in Channel 1 only the $y$-polarized mode is involved in the coupling as shown in Figs. 3(a) and 3(b). Hence, there are two loss peaks for the $y$-polarized mode and only one for the $x$-polarized mode.

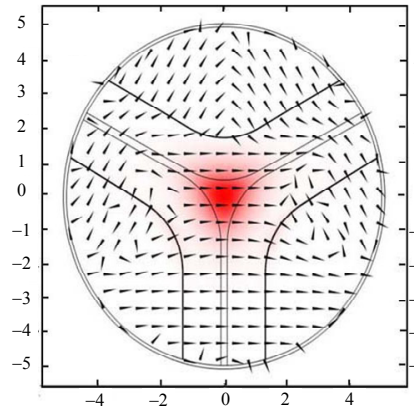

(a)

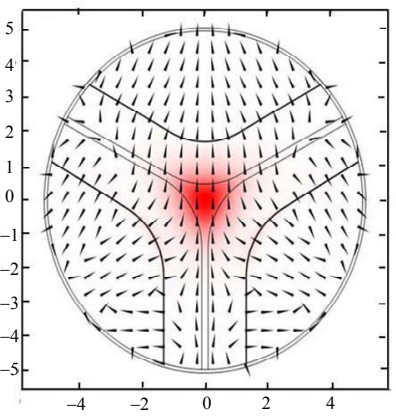

(b)
Fig. 3 Modes of three-strut wagon-wheel fiber shown in Fig. 1: (a) the $x$-polarized modes and (b) the $y$-polarized modes. Arrows show the direction of electric field in the $x-y$ plane of the fiber's cross section.

Another point should be added here to clarify the physics behind the resonance peaks for $x$ - and $y$-polarized fundamental modes. In the case, the refractive index of water 1.33 RIU is set as the reference in all three channels, and the refractive index profile has the $D_{3 \mathrm{~d}}$ symmetry. Substitution of (2) into (1) yields a two-wave system of equations for $x$ - and $y$-components of the electric field. Assuming $y$-axis to be along the line of symmetry in the refractive index profile, the Cartesian $x$-axis will be perpendicular to the axis of symmetry. In this setup, the $y$-polarized mode couples with the surface plasmons directly while a coupling between the $x$ polarized mode and $y$-polarized mode is necessary to be able to couple with the surface plasmons. Hence, 
the fundamental eigen-mode of the sensor with polarization in the $y$-direction is selected as the resonant mode in all cases investigated here, and the selection allows us to compare the present results with those of Zhang and coworkers [15].

\subsection{Thickness of the gold layer}

SPPs propagate along the interface between a gold layer and neighboring analyte, as the influence of dielectric is "sensed" by the traveling wave. Nano-scale properties of the gold layer, which are essential in the appearance of surface plasmon waves, are viable only if layer's thickness is between $20 \mathrm{~nm}$ and $50 \mathrm{~nm}$, beyond which aggregate properties will take hold [21]. Figure 4 shows the loss generated by exciting the fundamental $y$-polarized eigen-mode at different wavelengths for six different thickness values. In all six cases, struts' thickness is held at $150 \mathrm{~nm}$, and refractive index of the analyte filling all three channels is set at 1.33 RIU.



Fig. 4 Sensor's loss versus wavelength of input pulse for six different gold layer thickness values with analytes of refractive index equal to $1.33 \mathrm{RIU}$ in all channels and $t_{\mathrm{s} 1}=t_{\mathrm{s} 2}=150 \mathrm{~nm}$.

As can be seen from Fig. 4, double peaks appear for gold layer thickness values as low as $20 \mathrm{~nm}$ and as high as $40 \mathrm{~nm}$. The pattern reveals that the first resonant peak resulted from a pure coupling between the $y$-polarized mode and the surface plasmons increases while the second peak disappears. Disappearance of the second peak is the evidence of the weakening of the coupling between the $x$-polarized mode and the surface plasmons as the thickness of gold layer exceeds $30 \mathrm{~nm}$. Note that the first resonance peak, purely $y$-polarized peak, starts to decrease at the gold layer thickness of $35 \mathrm{~nm}$.
Since the $30 \mathrm{~nm}$ case shows a highest loss parameter in the first peak without losing the second peak. Hence, the gold layer is assumed to be $30 \mathrm{~nm}$ thick for the remaining parts of this investigation.

\subsection{Core struts' thickness}

Thickness of the core struts is the second parameter that can effectively alter the location and magnitude of resonant peaks. Figure 5 depicts changes in a loss parameter as a function of wavelength with respect to variations in the struts' thickness. Note that an increase in the struts' thickness leads to a decrease in the amplitude and left-shift of the loss parameter. This property can be utilized to separate the resonance peaks further. For all gold layer thicknesses in Fig. 5, the thickness of struts bounding Channel $1, t_{\mathrm{s} 1}$, is identical to the thickness of strut separating Channels 2 and 3, $t_{\mathrm{s} 2}$, as defined in Fig. 1.

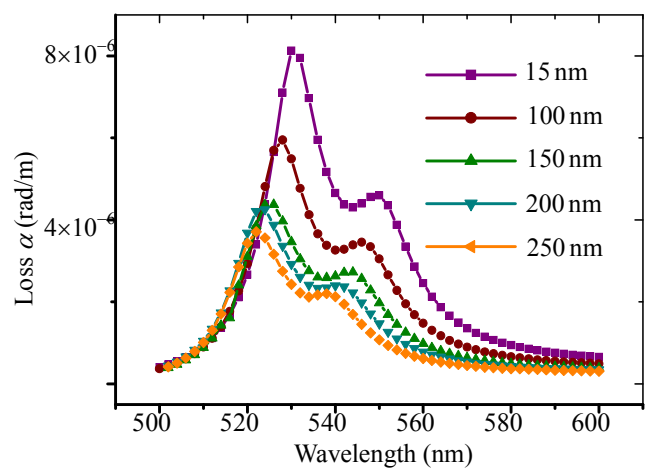

Fig. 5 Sensor's loss versus wavelength of the input pulse at 4 core struts' thickness values, case of $n=1.33$ RIU for analytes in all channels.

Building struts of the non-identical thickness allows for further separation of the two resonant peaks. Note that the symmetry of refractive index profile changes "slightly" from $D_{3 \mathrm{~d}}$ to $C_{2 \mathrm{v}}$, which in turn changes the degeneracy present in the fundamental mode of the wagon-wheel type sensor and also changes the eigenvalues. This would result in a slightly different values for the maximum wavelength and loss parameter, and consequently in separation of the peaks.

The two non-identical-struts cases are compared with the identical-struts case shown in Fig. 6, where the struts' thicknesses are $15 \mathrm{~nm}$. The two resonant peaks separate significantly, as shown in Fig. 6, 
suggesting that when non-identical-struts are used the resolvability of resonant peaks increases. Further, Fig. 6 shows that smaller $t_{\mathrm{s} 1}$ values lead to higher peaks loss values.

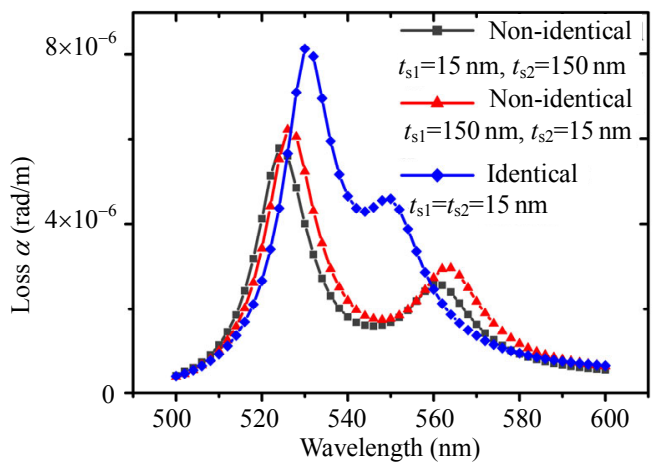

Fig. 6 Sensor's loss versus input pulse wavelength for identical and non-identical strut thickness values.

\subsection{Collective effect of the gold layer and struts' thickness}

To find the relation between the thickness of the thin layer of gold and the core struts' thickness, we investigate two cases. In the first case, the thickness of all struts is assumed to be $240 \mathrm{~nm}$, and the refractive index in all channels is set as 1.33 RIU. Thus, by varying the thickness of the gold layers from $23 \mathrm{~nm}$ to $40 \mathrm{~nm}$, a loss profile is obtained versus wavelength as shown in Fig. 7. The loss profile versus wavelength has one peak for the gold layer of $30 \mathrm{~nm}$ and higher due to SPR, while there are two peaks for the gold layer of $27 \mathrm{~nm}$ or less for struts' thickness of $240 \mathrm{~nm}$.

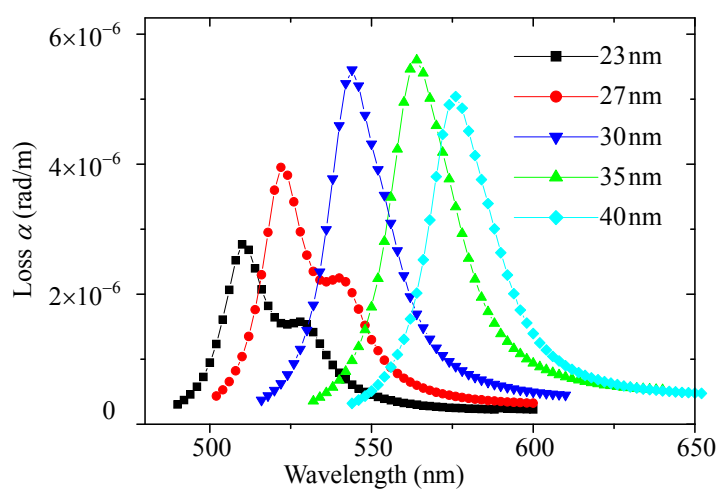

Fig. 7 Sensor's loss versus wavelength of input pulse for six different gold layer thickness values with analytes of refractive index equal to $1.33 \mathrm{RIU}$ in all channels and $t_{\mathrm{s} 1}=t_{\mathrm{s} 2}=240 \mathrm{~nm}$.

Comparing the result of Fig. 7 with that of Fig.4, one observes that by increasing the thickness of the struts, the presence of two-peak profile requires a thicker gold layer. The next case is shown in Fig. 8, where the loss profile versus wavelength is calculated for the non-identical strut thicknesses of $t_{\mathrm{s} 1}=15 \mathrm{~nm}$ and $t_{\mathrm{s} 2}=150 \mathrm{~nm}$ and the gold thickness of $23 \mathrm{~nm}$ to $40 \mathrm{~nm}$.

It is evident from Fig. 8 that when the $D_{3 \mathrm{~d}}$ symmetry breaks, the two peaks in $y$-polarized mode remain up to the gold thickness of $40 \mathrm{~nm}$. However, the two peaks are separated by $40 \mathrm{~nm}$ at gold thickness of $23 \mathrm{~nm}$, and they change just into a shoulder at gold thickness of $40 \mathrm{~nm}$.

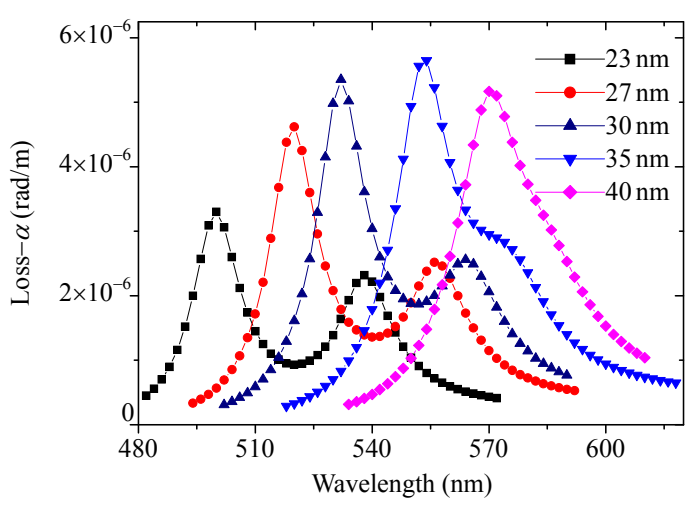

Fig. 8 Sensor's loss versus wavelength of input pulse for six different gold layer thickness values struts $t_{\mathrm{s} 1}=15 \mathrm{~nm}$ and $t_{\mathrm{s} 2}=$ $150 \mathrm{~nm}$.

\subsection{Sensor's length}

As the sensor is assumed two dimensional and the modes of the two dimensional cross sections are calculated, we believe that the pulse shape and the separation of peaks do not change. Hence, the sensor's length has no effect on the prediction of resonant peaks. A larger sensor's length will increase the absorption of plasmon waves thereby increasing the sharpness of the resonant peaks. The effect of the sensor's length on the loss curves can be deduced by the factor of $\alpha^{-1}$. As may be seen from Fig. 9, depending on sensor's length, power output decreases sharply at resonant peaks predicted by the corresponding loss curve in Fig. 6. Large sensor length can facilitate the process of fabrication, which also allows for a better interaction with the sample resulting in a more accurate sensor.

Based on the findings presented here, we propose a wagon-wheel type optical fiber sensor, as 
shown in Fig. 1, with a $30 \mathrm{~nm}$ thick gold layer and strut thickness values of $t_{\mathrm{s} 1}=150 \mathrm{~nm}$ and $t_{\mathrm{s} 2}=15 \mathrm{~nm}$. Although the length of sensor is not a factor in predicting the position of resonant peaks, however, we propose a sensor length of one half to one centimeter to provide sharp and clear pulses for better detection of analyte.

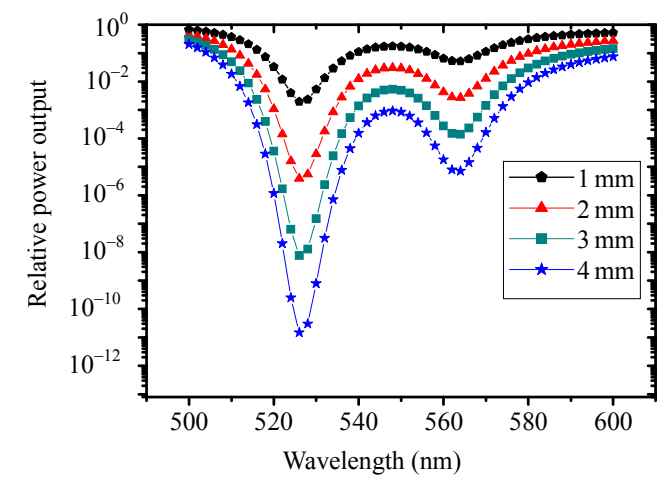

Fig. 9 Output power for sensor lengths of $1 \mathrm{~mm}, 2 \mathrm{~mm}, 3 \mathrm{~mm}$, and $4 \mathrm{~mm}$ containing similar analytes of $n=1.33$ RIU in all channels.

\subsection{Sensitivity and resolution of the sensor}

In the followings, sensitivity and resolution of the proposed sensor are investigated. Let the sensitivity of the sensor be defined as

$$
S=\frac{\Delta \lambda_{\text {peak }}}{\Delta n_{a}} \equiv \frac{\delta \lambda_{\text {peak }}}{\delta n_{a}}
$$

where $\Delta \lambda_{\text {peak }}$ is the change in the peak resonance wavelength for a change of $\Delta n_{a}$ in the refractive index of analyte $[5,15]$. Let the ratio of the instrumental wavelength detection limit $\Delta \lambda_{\min }$ over the sensitivity of the sensor defined as

$$
R=\frac{\Delta \lambda_{\min }}{S}
$$

be the refractive index resolution of the sensor [14], which is going to be simply called resolution.

In order to examine the resolution of the proposed sensor, a series of numerical tests are conducted. In each of the tests, all three channels are assumed to be filled with the same material, while corresponding loss parameters are plotted as a function of wavelength and presented in Fig. 10. Since this sensor is most suitable for bio-applications, defining water as the reference material ( $n=1.33 \mathrm{RIU}$ ) would be a suitable choice while assessing sensor's sensitivity and resolution.
The shift with respect to references' resonant, water, and peaks identifying the analyte that fills in all three channels are measured and shown in Fig. 10. Note that resonant peaks shift to the right, anywhere between $10 \mathrm{~nm}$ to $50 \mathrm{~nm}$ depending on the change in the testing analyte's refractive index. The first and second peaks are tabulated in Table 1 for different analytes' refractive indexes.



Fig. 10 Sensor's loss versus input pulse wavelength for cases of similar analytes in all three channels of the proposed sensor.

Making use of definition (7), we would have three different measures for the sensor's sensitivity. The first and second resonance peaks show a sensitivity of $1120 \mathrm{~nm} / \mathrm{RIU}$ and $1540 \mathrm{~nm} / \mathrm{RIU}$, respectively, while a different sensitivity is calculated based on the change in the two peak differences with respect to the change in refractive index, as given in Table 1, with a value of $420 \mathrm{~nm} / \mathrm{RIU}$. The third definition agrees with the definition made by Zhang et al. [15], which was claimed to be background free sensitivity.

Cennamo et al. [12] measured and calculated the refractive index sensitivity for a plastic optical fiber, specifically designed for biosensing applications, to be close to $2.5 \times 10^{3} \mathrm{~nm} / \mathrm{RIU}$. The structure of the fiber used by Cennamo and coworkers leads to wide transmittance lines as being compared with the wagon-wheel structure studied in the present work. Another D-shaped photonic crystal fiber was designed on the basis of SPR sensor technology by Chen et al. [13] which resulted in a similar sensitivity as that of [12]. Wagon-wheel geometry sensor was also designed and studied by Haukakorpi 
et al. [14] which resulted in the same sensitivity and resolution range as the present work. If the instrumental wavelength detection limit $\Delta \lambda_{\min }$ is assumed to be $0.1 \mathrm{~nm}$, the refractive index resolution is calculated to be $9 \times 10^{-5}$ RIU when the first peak is used for sensing. The result deduced here is comparable with the results given in [14]. Calculated values of the refractive index resolution of the sensor are given in Table 1 .

Table 1 First and second peaks in the absorption resonance for different refractive indexes. The sensitivity and resolution of the sensor are calculated when $\Delta \lambda_{\min }=0.1 \mathrm{~nm}$ is assumed.

\begin{tabular}{cccc}
\hline $\begin{array}{c}\text { Analyte's } \\
\text { refractive index }\end{array}$ & $\begin{array}{c}\text { First peak } \\
(\mathrm{nm})\end{array}$ & $\begin{array}{c}\text { Second peak } \\
(\mathrm{nm})\end{array}$ & $\begin{array}{c}\text { Peak diff. } \\
(\mathrm{nm})\end{array}$ \\
\hline 1.33 & 526 & 564 & 38 \\
1.34 & 536 & 576 & 40 \\
1.35 & 546 & 592 & 46 \\
1.36 & 560 & 610 & 50 \\
\hline$S(\mathrm{~nm} / \mathrm{RIU})$ & 1120 & 1540 & 420 \\
$R(\mathrm{RIU})$ & $9 \times 10^{-5}$ & $6.5 \times 10^{-5}$ & $2.4 \times 10^{-5}$ \\
error $\%$ & $0.22 \%$ & $0.39 \%$ & $1.6 \%$ \\
\hline
\end{tabular}

Analysis of the calculated data indicates high sensitivity even for the case close to the reference analyte. Furthermore, the sensitivity associated with the second peak is higher than the sensitivity associated with the first peak, indicating that the second peak moves faster than the first one in all cases. Hence, sensitivity expressed by the second peak is higher than the sensitivity expressed by the first peak. The similar trend is reported in [15]. However, the change in the sensitivity of the second peak with respect to peak one is larger in our case as compared with the values of reference [15].

A final set of test cases is simulated to determine the limits of the proposed sensor. While holding the same reference material in Channel 1, the refractive index for the analyte in Channels 2 and 3 is changed in its thousandths and ten-thousandths order. Figure 11 shows the loss profile for this test set, while Table 2 lists the calculated sensitivity and resolution of the sensor. It should be noted that the position of the first peak (the most intense peak) does not change although the symmetry of the detector changes slightly from $D_{3 \mathrm{~d}}$ to $C_{2 \mathrm{v}}$. The fact is that one of the peaks does not move as the analyte's refractive indexes in two channels change, which is a perfect choice as a sensor.

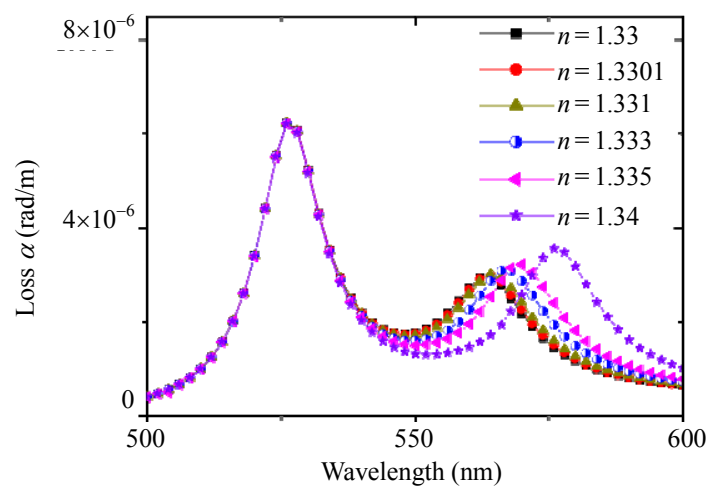

Fig. 11 Sensor's loss with respect to input pulse wavelength for cases with thousandth and ten-thousandth changes in the refractive index values.

Table 2 Sensitivity and resolution for analytes in Channels 2 and 3 .

\begin{tabular}{cc}
\hline Analyte's refractive index & $\lambda_{\text {peak }}(\mathrm{nm})$ \\
\hline 1.33 & 564 \\
1.331 & 564.5 \\
1.333 & 566 \\
1.335 & 570 \\
1.340 & 576 \\
Sensitivity (nm/RIU) & $1252+8 \%$ \\
Resolution (RIU) & $8 \times 10^{-5}$ \\
\hline
\end{tabular}

The sensitivity of the sensor when a reference is kept unchanged in the first channel and the second resonant peak with respect to the refractive index of analytes in Channels 2 and 3 is calculated to be $1252 \mathrm{~nm} /$ RIU. Hence, we can state that sensitivity of the proposed sensor is such that clear reading is possible even when the difference in refractive indexes of the analytes is within thousandths of one.

\subsection{Sensor tuning for analytes in two channels}

In this section, we show that it is possible to tune the sensor by choosing an appropriate thickness of struts. Zhang and coworkers [15] chose a coating layer over the gold layer in Channel 1 to tune the sensitivity for the analyte in Channels 2 and 3. The right thickness of the overlayer improves the sensitivity of the sensor.

We choose a different strategy for tuning the sensor. Figure 12(a) shows the diagrams depicting the proposed sensor's output power, when the gold layer is $30 \mathrm{~nm}$ thick and struts have the same 
thickness values, i.e., $t_{\mathrm{s} 1}=t_{\mathrm{s} 2}=150 \mathrm{~nm}$. The refractive index, $n_{a 1}$, for reference in Channel 1 is $1.33 \mathrm{RIU}$ while the refractive index, $n_{a 2}$, for the analyte in Channels 2 and 3 is changed from 1.33 RIU to 1.36RIU. As can be seen from Fig. 12(a), the output power as a function of input signal wavelength has two dips that move apart as the analyte's refractive index varies with respect to that of the reference material in Channel 1. More importantly, when the struts' thickness values are not identical, i.e., $t_{\mathrm{s} 1}=$ $150 \mathrm{~nm}$ and $t_{\mathrm{s} 2}=15 \mathrm{~nm}$, the two dips become more pronounced as well, which are clear from Fig. 12(b). The well pronounced separation of resonance peaks due to the difference in strut thickness did not appear in the work published by Zhang and coworkers [15]. Hence, the proposed sensor offers accurate and well resolved signals coming from

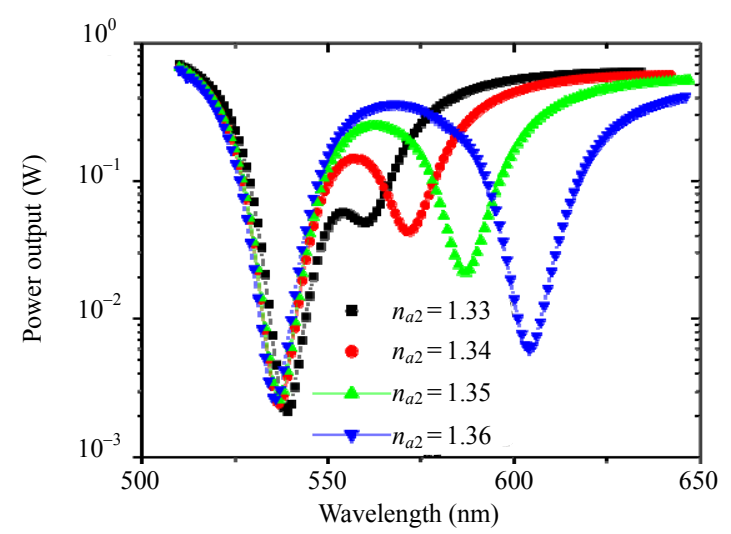

(a)

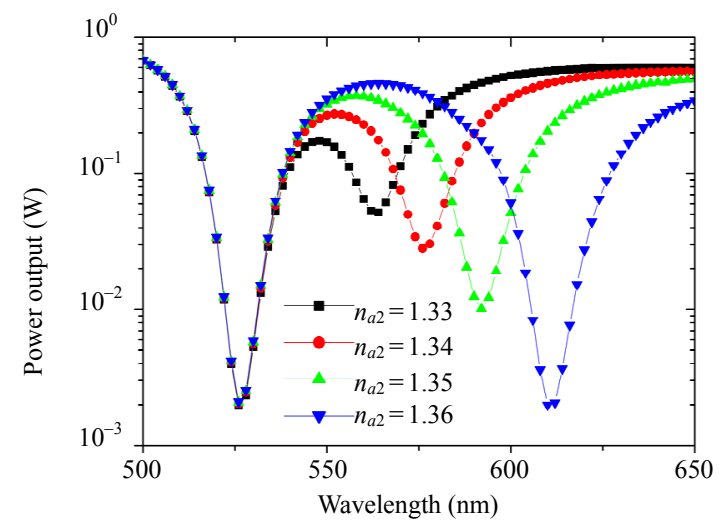

(b)

Fig. 12 Power output of sensor vs wavelength for $n_{a 1}=$ 1.33 RIU and $n_{a 2}=1.33 \mathrm{RIU}, 1.34 \mathrm{RIU}, 1.35 \mathrm{RIU}$, and 1.36 RIU in $y$-polarization for gold layer thickness of $30 \mathrm{~nm}$ : (a) for identical struts' thickness $\left(t_{\mathrm{s} 1}=t_{\mathrm{s} 2}=150 \mathrm{~nm}\right)$ and (b) for non-identical struts' thickness $\left(t_{\mathrm{s} 1}=150 \mathrm{~nm}, t_{\mathrm{s} 2}=15 \mathrm{~nm}\right)$. analytes which can be within a very small range in the refractive index, and makes this sensor a highly applicable tool in medical industry.

\section{Conclusions}

An optical fiber based SPR sensor using the three-struts wagon-wheel structure has been designed and analyzed for utilization in bio-industries, which is suitable for use in vivo or in vitro medical applications. The effective mode index $n_{\text {eff }}$ of the fundamental mode is calculated over the wavelength range of $500 \mathrm{~nm}$ to $650 \mathrm{~nm}$. Imaginary part of computed effective mode index is related to the absorption of light in the proposed wagon-wheel fiber structure. When all three channels are filled with the same analyte, symmetry element of the cross section is $D_{3 \mathrm{~d}}$. However, if there are two different materials in the channels, e.g. two analytes or a single analyte and a reference, the symmetry element changes to $C_{2}$. This results in further breaking the degeneracy of fundamental modes.

The thicknesses of the gold layer and the struts are selected to tune the proposed detector. It is found that $30 \mathrm{~nm}$ is the best thickness value for the gold layer, where the surface plasmon waves propagate. Furthermore, it is found that using different thickness values for the struts would separate and reduce the two resonant peaks coming from plasmon resonances in Channels 1, 2 and 3. Resolution for this sensor is calculated, and it is found that if the sensor is utilized for analytes with the refractive index close to that of water, then the closest discernable subject value could be within thousandths of the reference index, hence rendering it a highly sensitive optical sensor.

Final note should be added that the method used in this work is based on full vectorial finite element. This boundary value problem is solved by a home-written numerical algorithm called SyNA, which is also applicable in other fields $[17,18]$. The software is checked by comparing the results with other commercially available independent software. 


\section{Acknowledgment}

The authors would like to acknowledge the financial support, through a research grant, from Graduate University of Advanced Technology, Kerman, Iran.

Open Access This article is distributed under the terms of the Creative Commons Attribution 4.0 International License (http://creativecommons.org/licenses/by/4.0/), which permits unrestricted use, distribution, and reproduction in any medium, provided you give appropriate credit to the original author(s) and the source, provide a link to the Creative Commons license, and indicate if changes were made.

\section{References}

[1] E. Kretschmann and H. Raether, "Radiative decay of non-radiative surface plasmons excited by light," Zeitschrift für Naturforschung, 1968, 23(12): 2135-2136.

[2] A. Otto, "Excitation of nonradiative surface plasma waves in silver by the method of frustrated total reflection," Zeitschrift für Physik A Hadrons and Nuclei, 1968, 216(4): 398-410.

[3] J. Homola, H. Vaisocherova, J. Dostalek, and M. Piliarik, "Multi-analyte surface plasmon resonance biosensing," Methods, 2005, 37(1): 26-36.

[4] X. D. Hoa, A. G. Kirk, and M. Tabrizian, "Towards integrated and sensitive surface plasmon resonance biosensors: a review of recent progress," Biosensors and Bioelectronics, 2007, 23(2): 151-160.

[5] E. K. Akowuah, T. Gorman, S. Haxha, and J. V. Oliver, "Dual channel planar waveguide surface plasmon," Journal of the Optical Society of America , 2007, 24(6): 1423-1429.

[6] W. Peng, S. Banerji, Y. C. Kim, and K. S. Booksh, "Investigation of dual-channel fiber-optic surface plasmon resonance sensing for biological applications," Optics Letters, 2005, 30(22): 2988-2990.

[7] B. Špačková, M. Piliarik, C. Themistos, M. Rajarajan, and J. Homola, "Novel concept of multi-channel fiber optic surface plasmon resonance sensor," Sensors \& Actuators B Chemical, 2009, 139(1): 199-203.

[8] Z. Y. Zhang, P. Zhao, F. G. Sun, G. Z. Xiao, and Y. M. $\mathrm{Wu}$, "Self-referencing in optical-fiber surface plasmon resonance sensors," IEEE Photonics Technology Letters, 2007, 19(24): 1958-1960.

[9] Y. Wang, S. W. Meng, Y. Z. Liang, L. X. Li, and W. Peng, "Fiber-optic surface plasmon resonance sensor with multi-alternating metal layers for biological measurement," Photonic Sensors, 2013, 3(3): 202-207.

[10] J. Homola, I. Koudela, and S. S. Yee, "Surface plasmon resonance sensors based on diffraction gratings and prism couplers: sensitivity comparison," Analytical \& Bioanalytical Chemistry, 1999, 54(1): 16-24.

[11] J. Homola, S. S. Yee, and G. Gauglitz, "Surface plasmon resonance sensors: review," Sensors and Actuators B Chemical, 1999, 54(1-2): 3-15.

[12] N. Cennamo, D. Massarotti, L. Conte, and L Zeni, "Low cost sensors based on SPR in a plastic optical fiber for biosensor implementation," Sensors, 2011, 11(12): 11752-11760.

[13] Y. Z. Chen, Q. L. Xie, X. J. Li, H. S. Zhou, X. M. Hong, and Y. F. Geng, "Experimental realization of D-shaped photonic crystal fiber SPR sensor," Journal of Physics D Applied Physics, 2016, 50(2): 025101-025107.

[14] M. Hautakorpi, M. Mattinen, and H Ludvigsen, "Surface-plasmon-resonance sensor based on three-hole microstructured optical fiber," Optics Express, 2008, 16(12): 8427-8432.

[15] Y. T. Zhang, C. Zhou, L. Xia, X. Yu, and D. M. Liu, "Wagon-wheel fiber based multichannel plasmonic sensor," Optics Express, 2011, 19(23): 22863-22873.

[16] R. L. Olmon, B. Slovick, T. W. Johnson, D. Shelton, S. H. Oh, G. D. Boreman, et al., "Optical dielectric function of gold," Physical Review B Condensed Matter, 2012, 86(23): 235147-1-235147-9.

[17] A. R. Ahmadi, "SyNA, A general purpose finite element software system," Ph.D. dissertation Computational Research Center at Graduate University of Advanced Technology, Kerman, Iran, 2010.

[18] Z. Dehghanifard, A. R. Ahmadi, A. R. Ganjovi, and M. A. Bolorizadeh, "Space-time coupled finite element simulation of PECVD reactor," International Journal of Applied and Computational Mathematics, 2016, 2(3): 1-11.

[19] B. N Jiang, L. A. Povineli, and J. Wu, "The origin of spurious solutions in computational electromagnetics," Journal of Computational Physics, 1996, 125(1): 1597-1630.

[20] X. Yu, S. Y. Zahng, Y. Zhang, H. P. Ho, P. Shum, H. R. Liu, et al., "An efficient approach for investigating surface plasmon resonance in asymmetric optical fibers based on birefringence analysis," Optics Express, 2010, 18(17): 17950-17957.

[21] F. Mohr, Gold chemistry: applications and future directions in the life sciences. New York, USA: Wiley, 2009: 1-408. 